to the stretched lower end of the ileum, and tightly constricting the twisted colon. Had the tip of the appendix passed to its point of fixation in front of the cæcum, rotation of the latter must have taken place (as in several recorded cases) in the opposite direction.

The operative treatment of volvulus of the cæcum has proved more successful than might have been anticipated, close upon half the recorded cases having recovered. Untwisting, combined with fixation of the cæcum by at least two points to the parietes, is probably the best treatment, where the bowel is in good condition, but it may not be practicable until the viscus has been emptied by an incision, which is then carefully closed again. Excision of the affected parts may be required when gangrene or perforation is actually present, and where these conditions are threatening the temporary formation of an artificial anus at the cæcum may occasionally afford the safest means of relief.

Sheffield.

\section{THE SUCCESSFUL TREATMENT BY COLON LAVATION OF SOME CASES OF ECZEMA, PSORIASIS, URTICARIA, ACNE, AND PRURITUS.}

By ALFRED MANTLE, M.D. DURH., M.R. O.P. LOND., CONSULTING PHYSICIAN TO THE ROYAL HALIFAX INYIRMARY.

THERE is ample proof that in some individuals ingested toxins absorbed in the alimentary canal show the chief evidence of that absorption by changes in the skin. The most familiar case we recall and one easily demonstrable in some people is that of urticaria. In this instance the toxin is received from without and introduced into the system by some article of food. But it seems to me probable that urticaria, particularly when chronic, may also be due to auto-intoxication, the result of changes in metabolism of gastric, or more frequently, intestinal origin. Those cases of eczema which have been considered to be associated with gout ("gouty eczema") are likewise caused by a faulty metabolism, if we admit, as most of us do, that the symptomcomplex gout is due to a toxæmia of gastro-intestinal origin. Some people are born with an easily excited and irritable skin-a skin readily susceptible to internal and external pathogenetic stimuli or excitants. In such cases this predisposition may show itself in infancy and early childhood by an erythema or an eczema, which is corrected by attention to the gastro-intestinal canal, a fact which points to a toxæmic cause generated in that region.

A sensitive nervous system is commonly present in those subject to skin affections, and undoubtedly the condition of the mind influences very much the condition of the skin, and not infrequently we learn when taking the history of a case of eczema that the attack has been preceded by mental strain and anxiety. This is not difficult to explain, for no organs suffer more functional disturbance than those of digestion as a result of worry. A catarrhal condition of the mucous membrane of the stomach is not infrequent in such people, and if it becomes chronic loss of nervous energy and diminished muscular activity of the stomach may lead to its dilatation. Fermentative changes then take place, and the formation and absorption of toxins may follow. Bouchard believed auto-intoxication to be more common from a dilated stomach than the bowel even in the condition of constipation, and he noticed eczema as occurring in 13 out of 100 cases of gastric dilatation. ${ }^{1}$

But the benefit derived from colon lavation in certain cases of cutaneous eruptions suggests the skin disturbance to be more frequently associated with toxic absorption from the bowel than from the stomach, though doubtless the two absorptions are frequently associated.

Gastro-intestinal toxins seem to have a special affinity for the vaso-motor centres, thus resembling the toxins of malaria. And circulatory disorders are common with disturbances of the alimentary canal; therefore, it is not improbable that many cutaneous eruptions are due to irritation or stimulation of the vaso-motor centres by these toxins. It mu:t be remembered, however, that we all possess lines of defence

1 Trans. Bouchara Auto-intoxication in Disease, p. 162, 1894. against auto-intoxication, and it may be presumed that symptoms of toxæmia become manifest only when one or more of the defences (such as the cells of the intestinal mucosa and liver) become weakened and inefficient. This may happen when the intestinal epithelium is stripped by abrasions and ulceration (not uncommon in chronic constipation), or the liver or the kidneys become inadequate.

Eozcma.--This term covers a multiformity of cutaneous lesions due to a catarrhal inflammation of the skin, and onethird of the cases of skin disease fall into this category. For four or five years I have been making observations which have led me to the conclusion that disorders of the alimentary canal, and especially of the colon, must be given a high place in the etiology of many cases of eczema. Chronic constipation is common in such cases; and is frequently associated with a catarrhal condition of the mucous membrane of the colon in which the presence of mucus in the ejecta is very apparent. Mucus when normally secreted is invisibly mixed with the frees, but when seen in any quantity is abnormal, and frequently I have found after some experimental douches in cases of eczema, usually with, but at other times without, constipation, a considerable quantity of mucus, and treatment directed to this abnormality has resulted in the cure of the skin manifestation. I have therefore been led to connect the abnormal condition of the colon with the eczematous condition of skin, and to consider the latter as due to a toxamia of intestinal origin. When there is no skin eruption following a definite type with this condition of colon, there is usually a muddy appearance of the skin noticeable, and sometimes, in addition to this, an eczematous or other erupti $i n$.

A gentleman, aged 66 years, a free liver, was sent to me last October, when I found nearly the whole of the body affected with eczema. There was great pruritus, preventing sleep. The history pointed to a bowel infection, for, like not a few of these cases, there had been a local anal eczema and pruritus for a year or two, and the pruritus was so bad that an operation had been decided upon to relieve it. It had, however, been found by bacteriologial examination that the colon was unhealthy, and a course of intestinal lavation was decided upon. After each intestinal douche of alkaline sulphur water, which brought away considerable quantities of mucus, the patient had an immersion bath of a similar water, and he drank a stronger sulphur water each woorning. The improvement was very rapid both internally and externally, for as the colon became healthier and free from mucus the eczema and pruritus gradually disappeared. The patient returned home on the eighteenth day after treatment with no eruption, but he was advised to continue intestinal lavation at home for some time as a precautionary measure.

Other cases of eczema of a more local character have been treated equally successfully in the same way, and some of these have had a definite association with catarrhal inflammation of the bronchial tubes and asthma, and in other cases with gastric dilatation.

Psoriasis. - Whilst authors vary very much as regards the etiology of psoriasis, the late Dr. H. R. Crocker showed a connexion between it and digestive disorders, particularly after the age of 40 5ears. He says : " "Grout or its predisposing factors dyspepsia and constipation and alcoholism together made up half of thirty consecutive cases that I was consulted about." Stelwagon says of psoriasis: "Digestive and nutritive disturbances of all kinds are certainly provocative as to recurrences and of probable causative influences." 3 Pringle, Whitfield, and Brooke point to little or no connexion with gout, whilst the other authors quoted do; but arthropathies are mentioner by them as beirg seen with psoriasis, and rheumatoid arthritis is specially mentioned, which may be considered as frequently due to intestinal infection.

There is, as in the case of eczema, an inherited predisposition to the disease, but an external cause may provoke its development. In several cases when the symptoms and experimental douching have pointed directly to an intestinal causation the treatment already described has been brilliant in its results. In other cases with no particularly well-marked colitis good results have often, though not invariably, attended washing out the colon.

A lady, aged 57 years, unmarried, consulted me last year for psoriasis of 45 years' standing; there was inheritance in 
this case, for her mother had also suffered from it. She was of a nervous temperament and was much troubled with chronic constipation. The colon was considerably distended and the stomach somewhat dilated, and she was conscious of having passed mucus with the stools for years. She had been accustomed to come to Harrogate for many years and had derived a certain amount of benefit from drinking sulphur water and having sulphur baths. I ordered the colon to be washed out with 30 ounces of alkaline sulphur water and, as is the custom, this was repeated after all fæcal matter had been removed, and followed by an immersion bath of the same water. With a steady improvement in the condition of the bowel there was seen a decrease in the cutaneous eruption, and with three weeks' treatment the general condition was very satisfactory and better than it had been for years. I advised intestinal lavation to be continued at home, and only a few days ago I heard from this lady that she was "worlds better."

A very successful case with colitis was that of an army officer who had a history of psoriasis for many years. It was one with an unusually marked tendency to the gyrate and circinate variety on the trunk, whilst in the extremities the ordinary scattered, rounded patches were seen. The patient was very much better for his first course of treatment and was quite free from eruption after the second.

I have not had a large experience of this treatment in cases of psoriasis, but in every case I have treated in this manner there has been a very considerable improvement. I am inclined to think, however, that the large doses of arsenic which so many of these cases receive are detrimental in aggravating any catarrhal condition of the mucous membrane of the stomach and bowel.

Urticaria.-I have already stated that a chronic condition of urticaria may be kept up in certain individuals as the result of changes in metabolism producing toxins which after absorption bring about vascular changes in the skin. I am now excluding the poisons of shell-fish and drugs which are introduced from without. I have found the cause in two cases of urticaria to depend upon a toxæmia due to an abnormal condition of the mucous membrane of the stomach and colon.

A married lady, aged 37 years, with a hypersensitive nervous system and unstable circulation, was sent to me last year, having had periodic attacks of urticaria for a year which could not be traced to any article of diet. The history showed constipation and slight gastric disturbances for several years, and on investigating the condition of the colon mucous colitis was found to exist. A course of intestinal douches with deep massage of the abdomen, in order to give better tone to the intestinal wall, soon cleared up the mucous discharge, and brought about a better condition of peristalsis which has become permanent. No recurrence of the urticaria bas taken place since the treatment 12 months ago.

Another very similar case to this was that of a young married lady of much the same constitutional type, and in her case like treatment has been attended with equally successful results.

Aone. - A number of cases of acne vulgaris and of acne rosacea, the latter being in association with acne vulgaris, have been treated on these lines, and I agree with Whitfield in regarding seborrhcea as the primary cause of the acne disorder. Such patients are frequently of a nervous temperament, frequently suffer from gastric disorders and constipation, and instability of the peripheral circulation. Though some authorities (such as Bartholomy ${ }^{+}$) have frequently found dilatation of the stomach associated with acne, I have gene. rally found that an unhealthy condition of the mucous membrane of the colon is a greater source of the toxins, predisposing the skin to parasitic invasion, than is gastric dilatation. Intestinal lavation has been prescribed with success, but as in practically all these cases there is found a lack of proper attention to the scalp, this as well as the face must receive special attention.

A lady living in India and much exposed to weather consulted me last summer for acne rosacea. Her age was 42 years, she was gouty, and her circulation was poor. She had marked constipation, but bad but little digestive trouble. The eruption was more of the scaly papular kind than nodular, and without suppuration. There was an unhealthy

4 Étiologie et Thérapie de l'Acné. Archives Générales de Médecine, vol, il., 1889 , p. 641 condition of the scalp, and attention to this and the face, together with intestinal douching, caused a fairly rapid improvement, which I learn continues.

Similar treatment has been equally successful in two cases with suppurative nodules and acne vulgaris, and in other cases of acne simplex, and in some this was after vaccine treatment had failed to do good.

Pruritus. - The causes of pruritus are manv, but undoubtedly the nervous and gouty temperaments are specially favourable to its development; and in the majority of cases auto-intoxication the result of gastro-intestinal and hepatic disturbances is the leading cause of this distressing ailment. There is no primary lesion of the skin, but by scratching, which is difficult to avoid, eruptions do arise, and it must be borne in mind that pruritus is frequently the precursor of a well-recognised attack of eczema, lichen, or urticaria. It may be general, affecting more or less the whole body, or local, involving the anus, the vulva, the palms of the hands, or the soles of the feet. Very frequently there are marked vaso-motor disturbances, and in the case of a lady with pruritus confined chiefly to the lower extremities, and associated with mucous colitis, a very variable peripheral circulation existed.

With pruritus ani there is not infrequently a certain amount of eczema around the anus, and the rectal mucosa is often involved in the itching. In all cases where eczema has existed $I$ have found it to be the external and visible sign of a catarrhal condition of the mucous membrane of the colon, and internal douches of alkaline sulphur water, followed by immersion baths of the same water, have brought about such a marked change in the condition of the colon that the local skin lesions and irritation have soon cleared up

The method adopted in carrying out this special treatment by lavation for mucous colitis, associated or not with skin eruptions, is as follows. An alkaline sulphur water is generally used both for the intestinal douche and for the immersion bath which follows it. The object of the internal douche is to wash away old fæcal matter and mucus from

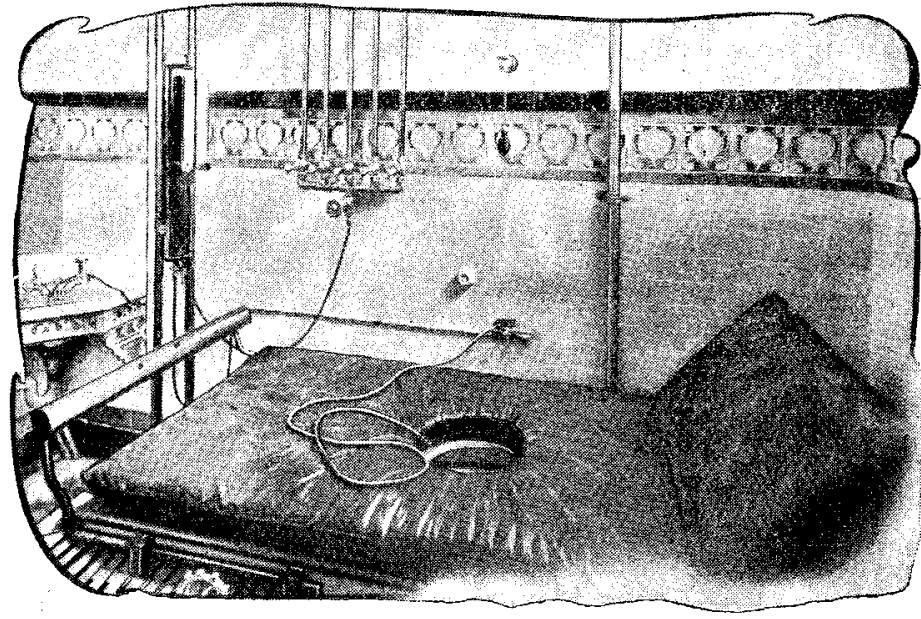

Internal douche: intestinal treatment.

the colon and to give the mucous membrane an antiseptic dressing. This is done in the following way. A long rubber tube, after being sterilised by boiling, is passed into the sigmoid and is attached to a hydrostatic douche, when from 20 to 40 ounces of sulphur water at a temperature of $105^{\circ} \mathrm{F}$. pass into the colon at a pressure of 2 feet, the patient lying first on the right side, then on the back, and lastly on the left side during the operation. This is repeated, and the ejecta after each douche are carefully examined and reported upon by a skilled attendant. Antiperistalsis of the colon normally exists, as pointed out by Hertz, 'and is an important factor in this treatment, enabling rectal injections to reach the ascending colon and cxcum when diseased. A warm immersion bath of sulphur water follows the internal douche, and when in this bath a hot douche at a higher temperature plays upon the wall of the abdomen under water from a large nozzle with fine perforations, and is chiefly directed over the site of the colon.

The immersion bath not only opens out the peripheral circulation, and thus relieves any congestion of the viscera s Brit. Med. Jour., vol. i., 1908, p. 193. 
which may exist, but is beneficial to the skin and nervous munifestations the result of auto-intoxication. I have shown before in these columns, when dealing with catarrhal and dilated conditions of the stomach, that in most of these cases there is a serious loss of balance in the circulation under the domain of the splanchnic nervous system in the causation of which toxins play an active part, for there is too much blood in the splanchnic area and too little in the periphery, and the restoration of the circulation is therefore all-important.

In all cases it is endeavoured to inake the treatment educational, and patients are advised to continue it at intervals after leaving Harrogate, but a second course of treatment is desirable in all chronic cases of colitis and skin disease if there is any tendency to recurrence.

Harrogate.

\section{CESAREAN SECTION UNDERTAKEN IN THE PRESENCE OF SEPTIC IN FECTION ;}

WITH A REPORT OF A CASE.

BY R. DRUMMOND MAXWELL, M.D. LOND., F. R. O.S. ENG.,

ORSTETRICAL TUTOR AND REGISTRAR TO THE LONDON HOSPITAL; PHYSICIAN TO OUT-WATIEN'S, (QUEEN CHARLOTTE'S IYLITG-IN GOSPIIAL, IIRYLEBONE ROAD, N.W.

ALL authorities agree that the maternal risks of Cæsarean section are increased directly by previous examinations unless conducted with the most scrupulous care; a few hold that previous obstetric manipulations or instrumentation definitely contra-indicate the operation where an alternative embryotomy is feasible. The morbidity and mortality of such infected cases are almost invariably brought about by soiling of the peritoneal cavity by an infected amniotic fluid, which in many cases, investigated post mortem, has been proved to be staphylococcic in nature. The sole risk, and it is no inconsiderable one, lies in this direction, and since it has been recognised a copious literature has sprung up on this subject in the last year or two, and fresh operations have been devised all with the object of preventing peritoneal inoculation by the selection of an extraperitoneal route and field of operation. It has been suggested that this should be attained by approaching the uterus from below the anterior parietal peritoneal reflection and by displacement of the bladder secure easy and safe access to the lower uterine segment. The criticism here is obvious-an incision is made into that part of the uterus least capable of retraction; the hæmorrhage that may ensue is difficult to check and has sometimes necessitated hysterectomy when the operator has gone "too low" even in the performance of the classical operation.

An alternative suggestion recommends opening the peritoneal cavity and in various ways " tenting" off the operation field by suturing the anterior parietal peritoneum to the uterus above the level of the proposed incision. Against this $I$ would advance the arguments that such suturing, to be accurate and above all watertight, will be almost impossible to carry out; while the time employed may be considerable -a most undesirable event in a Cæsarean section where a foetal factor has to be considered.

The strongest point that can be raised against the extraperitoneal technique and which equally applies to such alternatives as symphysiotomy and pubiotomy is, that in the presence of such an emergency as obstructed labour which not only the obstetric surgeon but the less practised accoucheur may have to face, it is imperative to reduce technique to the simplest form consistent with safety to the patient.

Have we then, if we reject the extraperitoneal technique, no alternative but to perform embryotomy in these doubtful cases? Surely, if the danger lies with the infected amniotic fluid, we can at least make an endeavour to get rid of it or reduce its toxicity, or failing that to prevent its access to the abdominal cavity.

Now, as regards attempts to wash out liquor amnii (or

6 The Influence of the Nerrous System and External Temperature upon Certain Circulatory Changes Concerned in the Etiology of Catarrh, Ulcer, and Simple Dilatation of the Stomach, THE LAACET, vol. i., 1906, p. 1031 . "after-waters," as they are in these cases) with the foetus still in utero, the difficulties are much greater than at first appears to be the case. Very little indeed is knuwn of the dynamics and hydrostatics of the second-stage uterus. Matthews Duncan, Labs, and Olshausen are the chief authorities we bave to rely on for intormation on these points.

Is there always a collection of "after-waters" capable of being irrigated after the patient has been in labour many hours, or does the uterine wall closely come in contact with the superficies of the child, reducing the upper uterine " cavity" to a merely potential space? One's own observa. tions made on Cresarean sections conducted on second-stage patients certainly do not support the view of Lahs, that "after-waters" are present to any appreciable extent. Most of these uterine incisions result in a perfectly "dry" extraction of the fcetus with no noticeable gush of amniotic fluid.

Now, if it be that there is no amniotic collection to wash out, the only means of irrigating the infected cavity, with the foetus in situ, will be by passing up a douche nozzle to the fundus and irrigating the cavity fretly with some aseptic neutral fluid. In the case I am about to describe this was effected by using a soft pewter douche-tube (a glass tube would be dangerous). The douch-tube was introduced by my assistant (Dr. H. Stevens, resident medical officer at the hospital), the tip being clearly $f \in l t$ at the fundus through the parietes. A copious flow of sterile salt solution was allowed to run, at a temperature of $105^{\circ} \mathrm{F}$., for a couple of minutes, and the flushing effect on the uterus was obvious. The laparotomy was performed as soon as the nozzle was withdrawn, and the uterus was "eventrated" and incised at once. It was remarked that the extraction of the child was perfectly "dry," although barely a minute before the fundus must have contained several ounces of salt solution. The report of the case briefly is as follows.

The patient, aged 30 years, I-gravida, was admitted to Queen Charlotte's Hospital on June 18th, 1910, at 6 P.M. She was markedly rachitic, with characteristic deformity of the lower extremities. The true conjugate was estimated at just under 3 inches. The patient's condition could not be considered "favourable" as labour had been in progress for 40 hours and the membranes were mptured. The head was "floating" above the brim and the uterus was temporarily almost inactive. There was a history of attempted forceps extraction: on examination there was no trauma of the cervix or lower genital tract. The pulse was 110, and the temperature was normal. The fotal heart-rate was normal and regular, between 120 and 130. There was, however, considerable maternal distress. In the presence of an appa rently unharmed foetus embryotomy was decided against the abdomen was painted with tincture of indine, while preparations for immediate Cæsarean section were carried out.

Prior to the section one hour later the abdomen was again painted with iodine and the uterine cavity washed out with sterile salt solution. The temperature was $105^{\circ} \mathrm{F}$. It was felt that at this temperature, which would be reduced inside the uterus to approximately $100^{\circ}$, no risks of provoking premature fcetal respiration would be caused. The head was displaced and the soft pewter douche nozzle was passed with ease to the fundus; several quarts of saline solution were washed through the uterus and escaped freely. An 8-inch incision was made in the parietes in the middle line, 4 inches above and 4 below the navel. The uterus was "eventrated" through the incision and the edges of the wound were carefully packed round with sterilised towels. A median sagittal fundal incision was made, the placenta being encountered at the lower end. The incision was practically bloodless and the foetus was easily extracted, with no apparent escape of fluid accompanying it. The uterine wound and abdominal incision were closed in the usual way, using stout and finer silkworm-gut sutures throughout. The patient was not sterilised.

The foetal head showed well-marked moulding and a forceps "grip" over the right frontal eminence. Some anxiety was felt for the child during the first 48 hours owing to pyrexia (maximum temperature $105^{\circ}$ ) very suggestive of the septic broncho-pneumonia to which so many of these children succumb (even in the absence of trauma) after long labour and exposure to infected liquor amnii. The child, however, made a rapid recovery and has thriven well on the breast, supplemented by the bottle owing to defective nipple 